\title{
When will we get a new class of analgesic agent based on animal study data?
}

\author{
Tatsuo Yamamoto
}

Received: 30 January 2014/ Accepted: 11 February 2014/Published online: 9 March 2014

(C) Japanese Society of Anesthesiologists 2014

Prostaglandins are known to be key to producing and maintaining the pain state [1]. It has been reported in animal studies that intrathecal injection of prostacyclin receptor agonist induced mechanical allodynia in an inflammatory pain model [2] and spinal cyclooxygenase (COX) inhibition by indomethacin-attenuated mechanical allodynia and increase in activated microglia in a neuropathic pain model [3]. Intrathecal injection of COX-2 selective inhibitor, but not COX-1 selective inhibitor, produced a good analgesic effect on inflammatory pain [4]. These data strongly suggested that, in the animal model, spinal prostaglandins synthesized by COX-2 have an important role in nociceptive transmission and maintenance of spinal hypersensitivity. In a clinical study, spinal administration of lysine acetylsalicylate was reported to produce an analgesic effect on chronic refractory pain in cancer patients [5], but intrathecal injection of ketorolac failed to reduce various experimental pain and acute postoperative pain $[6,7]$. The role of spinal prostaglandins in the maintenance of the pain and hypersensitivity states in humans is still unclear.

Wang et al. [8] reported, in this issue, that intrathecal injection of ketorolac did not reduce acute and chronic postoperative pain. This report suggested that spinal prostaglandins did not play an important role in the production and maintenance of the pain and hypersensitive state in a human clinical situation. As just mentioned, most of the animal studies demonstrated that spinal prostaglandins played an important role in pain transmission and maintenance of spinal hypersensitivity and that spinal COX

T. Yamamoto $(\bowtie)$

Department of Anesthesiology, Graduate School of Medical

Science, Kumamoto University, Kumamoto, Japan

e-mail: yamamotot@fc.kuh.kumamoto-u.ac.jp inhibitor elicited an analgesic effect, but most of the human studies showed that intrathecal injection of COX inhibitors produced no analgesic effect. There was always a large discrepancy between the results in animal pain studies and those in human pain studies. Many candidate analgesic drugs have been introduced from animal studies, but most of these drugs were not effective against clinical pain in humans.

Pain is always subjective. Although tissue damage may often be essential for the experience of pain in humans, pain is not necessarily dependent on it. In an animal study, we do not know how the animals feel pain. In an animal study, we can measure the nociceptive level using reflex response against nociceptive stimuli, but this result is not able to measure pain level. Recently, $\alpha 2$-subunit blockers, such as gabapentin and pregabalin, have been introduced for the treatment of neuropathic pain. Gabapentin was, at first, introduced as an anticonvulsant. Because some anticonvulsants were effective against neuropathic pain, clinicians used $\alpha 2 \delta$-subunit blockers for the treatment of neuropathic pain without any evidence and found a good antineuropathic pain effect by chance. When will we get a new class of analgesic agent based on animal study data?

\section{References}

1. Vanegas H, Schaible H-G. Prostaglandins and cycloxygenases in the spinal cord. Prog Neurobiol. 2001;64:327-63.

2. Doi Y, Minami T, Nishizawa M, Mabuchi T, Mori H, Ito S. Central nociceptive role of prostacyclin (IP) receptor induced by peripheral inflammation. Neuroreport. 2002;13:93-6.

3. Kunori S, Matsumura S, Okuda-Ashitaka E, Katano T, Audoly LP, Urade Y, Ito S. A novel role of prostaglandin E2 in neuropathic pain: blockade of microglial migration in the spinal cord. Glia. 2011;59:208-18. 
4. Yaksh TL, Dirig DM, Conway CM, Svensson C, Luo ZD, Isakson PC. The acute antihyperalgesic action of nonsteroidal, antiinflammatory drugs and release of spinal prostaglandin E2 is mediated by the inhibition of constitutive spinal cyclooxygenase- 2 (COX-2) but not COX-1. J Neurosci. 2001;21:5847-53.

5. Pellerin M, Hardy F, Abergel A. Chronic refractory pain in cancer patients: value of the spinal injection of lysine acetylsalicylate. Presse Med. 1987;16:1465-8.

6. Eisenach JC, Curry R, Tong C, Houle TT, Yaksh TL. Effects of intrathecal ketorolac on human experimental pain. Anesthesiology. 2010;112:1216-24.
7. Eisenach JC, Curry R, Rauck R, Pan P, Yaksh TL. Role of spinal cyclooxygenase in human postoperative and chronic pain. Anesthesiology. 2010;112:1225-33.

8. Wang L, Bauer M, Curry R, Anders L, Sessler DI, Eisenach JC. Intrathecal ketorolac does not improve acute or chronic pain after hip arthroplasty: a randomized controlled trial. J Anesth. 2014. doi:10.1007/s00540-014-1798-6. 